This item was submitted to Loughborough's Research Repository by the author.

Items in Figshare are protected by copyright, with all rights reserved, unless otherwise indicated.

\title{
Classical correlations of defects in lattices with geometrical frustration in the motion of a particle
}

\section{PLEASE CITE THE PUBLISHED VERSION}

http://dx.doi.org/10.1103/PhysRevB.73.174417

\section{PUBLISHER}

(C) The American Physical Society

\section{VERSION}

VoR (Version of Record)

\section{LICENCE}

CC BY-NC-ND 4.0

\section{REPOSITORY RECORD}

Pollmann, Frank, Joseph J. Betouras, and E. Runge. 2019. "Classical Correlations of Defects in Lattices with Geometrical Frustration in the Motion of a Particle". figshare. https://hdl.handle.net/2134/12776. 


\title{
Classical correlations of defects in lattices with geometrical frustration in the motion of a particle
}

\author{
Frank Pollmann, ${ }^{1}$ Joseph J. Betouras, ${ }^{1,2, *}$ and Erich Runge ${ }^{3}$ \\ ${ }^{1}$ Max-Planck-Insitut für Physik komplexer Systeme, Nöthnitzer Strasse 38, 01187 Dresden, Germany \\ ${ }^{2}$ Instituut Lorentz, Universiteit Leiden, Niels Bohrweg 2, 2333 CA Leiden, The Netherlands \\ ${ }^{3}$ Institut für Physik, Technische Universität Ilmenau, 98684 Ilmenau, Germany \\ (Received 23 February 2006; revised manuscript received 17 April 2006; published 17 May 2006)
}

\begin{abstract}
We map certain highly correlated electron systems on lattices with geometrical frustration in the motion of added particles or holes to the spatial defect-defect correlations of dimer models in different geometries. These models are studied analytically and numerically. We consider different coverings for four different lattices: square, honeycomb, triangular, and diamond. In the case of a hard-core dimer covering, we verify the existing results for square and triangular lattices and obtain new ones for the honeycomb and diamond lattices while in the case of a loop covering we obtain new numerical results for all the lattices and use the existing analytical Liouville field theory for the case of a square lattice. The results show power-law correlations for the square and honeycomb lattices, while exponential decay with distance is found for the triangular lattice and exponential decay with the inverse distance on the diamond lattice. We relate this fact to the lack of bipartiteness of the triangular lattice and in the latter case to the three dimensionality of the diamond. The connection of our findings to the problem of fractionalized charge in such lattices is pointed out.
\end{abstract}

DOI: 10.1103/PhysRevB.73.174417

PACS number(s): 05.50.+q, 71.10.-w, 71.27+a

\section{INTRODUCTION}

There has been enormous interest in the properties of quantum frustrated magnets, with recent studies showing fractionalization of quantum numbers (see Refs. 1 and 2, and citations therein). More recently, attention has shifted to charge degrees of freedom on lattices with frustrated geometries where the number of classical ground-state configurations increases exponentially with the number of sites. It has been noticed that hopping of fermions from site to site in frustrated lattices can lead, under certain conditions, to fractionalization of the fermionic charge in a rather natural way. 4,5

The motion of spinless fermions (or hardcore bosons) on a lattice can be mapped to an Ising spin problem or to a dimer configuration for any given lattice geometry and filling fraction as we will see in the next paragraph. The addition of a fermion (or boson) leads to a flip of an Ising spin or, equivalently, to a new dimer. The ground states of the considered systems fulfill a local constraint of having a certain number of particles on each unit cell or, equivalently, a certain number of dimers at each site.

For a specific implementation of local constraints in the Hamiltonian language for fermions, let us consider the model Hamiltonian

$$
H=-t \sum_{\langle i j\rangle}\left(c_{i}^{\dagger} c_{j}+\text { H.c. }\right)+V \sum_{\langle i j\rangle} n_{i} n_{j} .
$$

Here $\langle i j\rangle$ denotes the sum over nearest neighbors $i$ and $j$. The operators $c_{i}\left(c_{i}^{\dagger}\right)$ annihilate (create) a particle on site $i$, and $n_{i}=c_{i}^{\dagger} c_{i}$. For a moment, let us set the hopping integral $t$ to zero. We are interested in lattices for which the ground state of the Hamiltonian (1) with repulsive nearest-neighbor interaction term $V$ only has at certain fillings a macroscopic degeneracy, which increases exponentially with the system size. In other words, the system has a finite $T=0$ entropy (for possible technical applications, see Ref. 6). Examples of such lattices are the pyrochlore lattice and the crisscrossed checkerboard lattice (two-dimensional pyrochlore), shown as Figs. 1(a) and 1(b). At half filling, all classical ground states fulfill the so-called tetrahedron rule of having exactly two particles on each tetrahedron (crisscrossed square). ${ }^{3}$ The dimer coverings of the dual lattice are constructed as usual by connecting all centers of the tetrahedra and drawing a dimer whenever the traversed site of the original lattice is occupied. The tetrahedron rule translates into the constraint of having exactly two dimers at each site of the dual lattice. Similarly, for the kagomé lattice with nearest-neighbor repul-

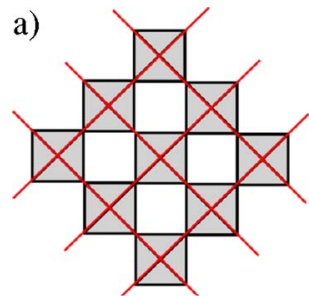

b)

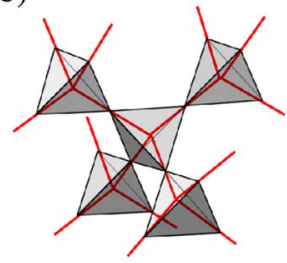

c)

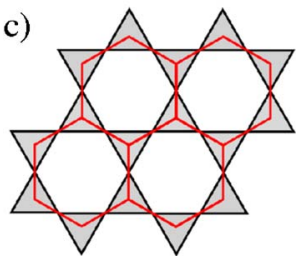

d)

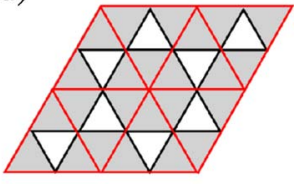

FIG. 1. (Color online) Mapping of lattices which show frustration (black) to dual lattices (red). (a) Checkerboard lattice with nearest-neighbor repulsion $\rightarrow$ square lattice, (c) pyrochlore lattice with nearest-neighbor repulsion $\rightarrow$ diamond lattice (c) kagomé with nearest-neighbor repulsion $\rightarrow$ honeycomb lattice, and (d) kagomé with repulsion interaction on hexagons only $\rightarrow$ triangular lattice. The constraint of having a certain number of particles on each unit cell translates into the constraint of having a fixed number of dimers touching each site of the dual lattice, leading to a hard-core dimer or loop dimer covering. 


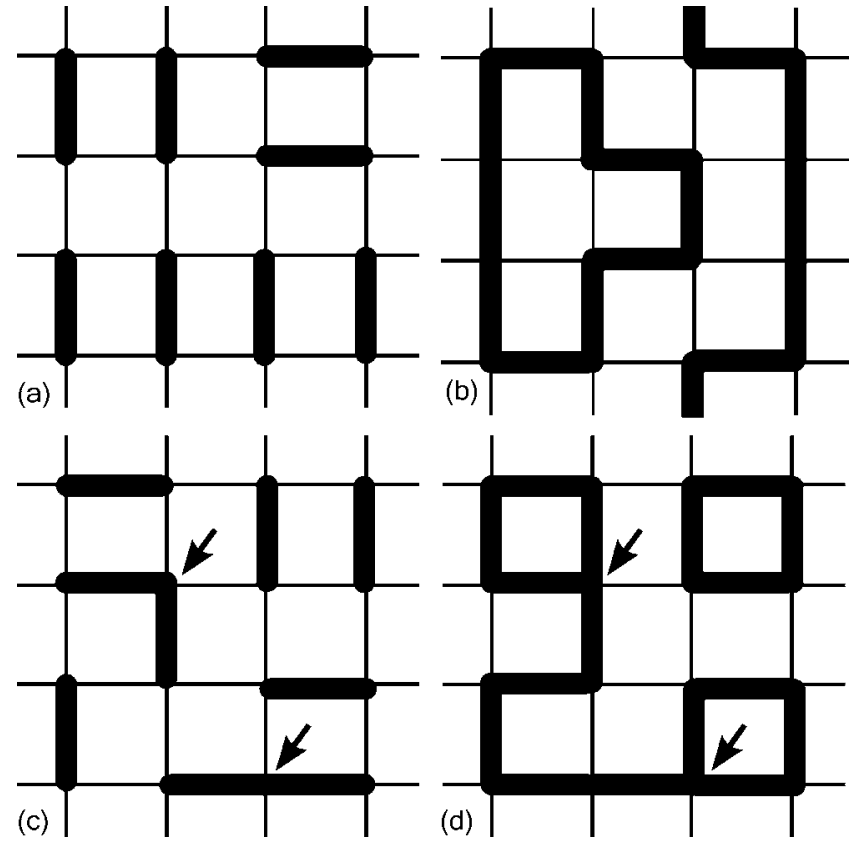

FIG. 2. Hard-core dimer (a) and loop dimer (b) covering on the square lattice. (c) and (d) show two defects in the different coverings at positions marked by arrows.

sion the dual lattice is the honeycomb; see Fig. 1(c). If the repulsive interaction on the kagomé lattice is restricted to hexagons only, the dual lattice is a triangular lattice; see Fig. 1(d).

In the long run, one wants to understand the behavior of mobile, fractionally charged, excitations resulting from weak particle or hole doping of such systems.

As a first step, we present the study of classical defectdefect correlations. A defect here means a cell where the rule of a fixed number of particles (or dimers) is violated (Fig. 2). The study includes bipartite and nonbipartite two-dimensional lattices (checkerboard, honeycomb, and triangular lattices) as well as a three-dimensional diamond lattice, with two different fillings corresponding to (i) the loop dimer and the (ii) hard-core dimer covering. Wherever possible, we obtain analytical results and compare them to numerical simulations. These classical correlation provide information about correlations near the Rokshar-Kivelson (RK) point of the quantum Hamiltonian because at the RK point the quantummechanical ground state is given by an equal-weighted superposition of all configurations. ${ }^{7}$ Furthermore, in our case, one can expect classical correlations to hold more generally since the low-energy excitations can be described equivalently by hard-core bosons or fermions. ${ }^{8}$

Historically, studies of monomer and dimer models started very early. ${ }^{9}$ At zero doping, the Pfaffian method introduced by Kasteleyn allows the derivation of the classical correlations analytically. ${ }^{10}$ For the square lattice with hardcore dimer covering, the correlation of a pair of monomers has been obtained. ${ }^{11,12}$

The main findings of the present work are the following: For the square and the honeycomb lattice, correlations as a function of distance show a power-law behavior, with an exponent $1 / 2$ for the hard-core dimer covering on the square lattice and $1 / 3$ for a loop dimer covering. The honeycomb lattice has an exponent of $1 / 2$ for both coverings. An exponential decay with distance is found for the triangular lattice, and the diamond lattice shows exponential decay with inverse distance.

In the next section, we present the results and the methods in detail. We start with the square lattice where, for completeness, we summarize the Liouville theory of the fully packed loop model with two colors, ${ }^{13}$ which provides the exponent for the loop covering analytically, and we subsequently show the numerical results. We then present the relevant results for the honeycomb, the triangular, and the diamond lattices and, finally, we conclude.

\section{RESULTS FOR DIFFERENT LATTICES}

\section{A. Square lattice}

As described above, the half-filled checkerboard lattice with nearest-neighbor repulsion is mapped to a loop dimer model on the square lattice, meaning that each site is connected to exactly two dimers. This can then be interpreted as covering in the fully packed loop model with two colors, assigning different colors to occupied and unoccupied bonds. Using the machinery developed for this model, ${ }^{13}$ we can read off the appropriate exponent of the defect-defect correlation function: In order to obtain the Liouville theory of the model, the first step is to map the oriented loops to an interface model, where they can be interpreted as contour lines. The microscopic heights are defined at the center of the lattice plaquettes, and each bond is in one of four possible states $(\vec{A}, \vec{B}, \vec{C}, \vec{D})$. We write the partition function as $Z$ $=\Sigma_{G} n_{b}^{N_{b}} n_{g}^{N_{g}}$ where $N_{b}$ and $N_{g}$ are the numbers of the loops of the two colors and $0 \leq n_{b}$ and $n_{g} \leq 2$ are the fugacities accordingly. Assigning weights $\exp \left( \pm i \pi e_{b}\right)$ or $\exp \left( \pm i \pi e_{g}\right)$ to the different orientations, with $n_{b}$ $=2 \cos \left(\pi e_{b}\right)$ and $n_{g}=2 \cos \left(\pi e_{g}\right)$, allows a local definition of the statistical weight of oriented loop, and in turn the weights leads to a local field theory. ${ }^{13}$

Our problem at hand (which can be equivalently mapped to a six-vertex model) corresponds to fugacities $n_{b}=n_{g}=1$. Therefore $e_{b}=e_{g}=1 / 3$. Adopting the same convention as in Ref. $10[\vec{A}=(-1,+1,+1), \vec{B}=(+1,+1,-1), \vec{C}=(-1,-1,-1)$, $\vec{D}=(+1,-1,+1)]$, the topological charge $( \pm \vec{m})$ is $\vec{m}=\vec{C}-\vec{A}$ $=(0,-2,-2)$ and $2 x_{1,1}$ is the dimension of an operator with total charge $\left(\vec{e}_{0}, \vec{m}_{1,1}\right)$, where $\vec{e}_{0}=-\pi / 2\left(e_{g}+e_{b}, 0, e_{g}-e_{b}\right)$ $=\pi(1 / 3,0,0)$. The effective field theory for the coarsegrained heights is given by the action

$$
\begin{gathered}
S=S_{E}+S_{B}+S_{L}, \\
S_{E}=\frac{1}{2} \int d^{2} x K_{\alpha, \beta} \partial h^{\alpha} \partial h^{\beta}, \quad S_{B}=\frac{i}{4 \pi} \int d^{2} x\left(\vec{e}_{0} \cdot \vec{h}\right) \mathcal{R}, \\
S_{L}=\int d^{2} x \sum_{\vec{e} \in R_{\omega}^{*}} \widetilde{\omega}_{\vec{e}} \exp [i \vec{e} \cdot \vec{h}(x)] .
\end{gathered}
$$

Here, $S_{E}$ is the elastic term (entropy of the oriented loops) with the tensor $\mathcal{K}$ of elastic constants $K_{11}=K_{33}$ and $K_{12}$ 
$=K_{23}=0$ and $\vec{h}(\vec{x})$ is the height field (coarse-grained field of the integer-valued height $h$ ). $S_{B}$ is the boundary term with the scalar curvature $\mathcal{R}$, which vanishes everywhere except at the boundary. This term inserts the vertex operator at the far ends and supplies winding loops. Finally, $S_{L}$ is the Liouville term. The Liouville potential is the coarse-grained version of the local weight assigned to an oriented loop configuration $\Pi_{\vec{x}} \lambda(\vec{x}): \omega(\vec{x})=-\ln [\lambda(\vec{x})]$ is defined in height space, which is the bcc lattice $R_{\omega}$. The Fourier-transformed values of $\omega(\vec{x})$ $=\Sigma_{\vec{e} \in R_{\omega}^{*}} \widetilde{\omega}_{\vec{e}} \exp [i \vec{e} \cdot \vec{h}(x)]$ in the reciprocal lattice $R_{\omega}^{*}$ (a fcc lattice) enter $S_{L}$.

The scaling dimension of a general operator with both electric and magnetic charge is given by

$$
x(\vec{e}, \vec{m})=\frac{1}{4 \pi}\left[\vec{e} \cdot \mathcal{K}^{-1} \cdot\left(\vec{e}-2 \vec{e}_{0}\right)+\vec{m} \cdot[\mathcal{K} \cdot \vec{m}]\right],
$$

where in our case $K_{11}=\frac{\pi}{8}\left(2-e_{b}-e_{g}\right)=\frac{\pi}{6}, K_{13}=\frac{\pi}{8}\left(e_{b}-e_{g}\right)=0$, and

$$
K_{22}=\frac{\pi}{2} \frac{\left(1-e_{b}\right)\left(1-e_{g}\right)}{2-e_{b}-e_{g}}=\frac{\pi}{6} .
$$

Therefore the critical exponent of interest is simply

$$
\begin{aligned}
2 x_{1,1}= & \frac{1}{4}\left[\left(1-e_{b}\right)+\left(1-e_{g}\right)\right]+\frac{\left(1-e_{b}\right)\left(1-e_{g}\right)}{2-e_{b}-e_{g}} \\
& -\left[\frac{e_{b}^{2}}{1-e_{b}}+\frac{e_{g}^{2}}{1-e_{g}}\right]=\frac{1}{3} .
\end{aligned}
$$

The result (3) will be next verified numerically by Monte Carlo simulations.

The dimer models on dual lattices are numerically preferable over the original lattices, because the constraints are included in a more natural way. Even though dimer models have been intensively investigated in the study of spin models, ${ }^{2}$ not much is known about defect-defect correlations. The defect-defect correlation function, e.g., $C(\mathbf{0}, \mathbf{r})$ $\sim\left\langle N_{\mathbf{0}} N_{\mathbf{r}}\right\rangle$, which gives the probability to find two defects at a certain distance $\mathbf{r}$. The defect-defect correlation is proportional to the restricted partition function $Z_{11}(\mathbf{0}, \mathbf{r}) \sim|\mathbf{r}|^{-2 x_{11}}$ of the two-color fully packed loop model. It is counting the number of configurations with defects at $\mathbf{0}$ and $\mathbf{r}$ connected by one string of each color. Thus $C(\mathbf{0}, \mathbf{r})$ is expected to become isotropic at large distances and to decay algebraically $Z(\mathbf{0}, \mathbf{r}) \sim|\mathbf{r}|^{1 / 3}$.

In the following, we measure numerically the correlations along a coordinate axis $[\mathbf{r}=(x, 0)]$ and refer to it as $C(x)$. The classical two-point correlation functions at zero temperature are delivered as averages over all degenerate ground states. The number of degenerate ground states grows exponentially with system size. At the start, an allowed configuration with fixed filling; i.e., a ground state of the undoped system with no violation of any local constraint is generated. Then we add a dimer onto an unoccupied random link. This leads to two defects on adjacent sites of the dual lattice, which subsequently propagate via local dimer moves through the system without creating any new defects. At each step the defect-defect distance $x$ is counted in a histogram $Z(x)$,

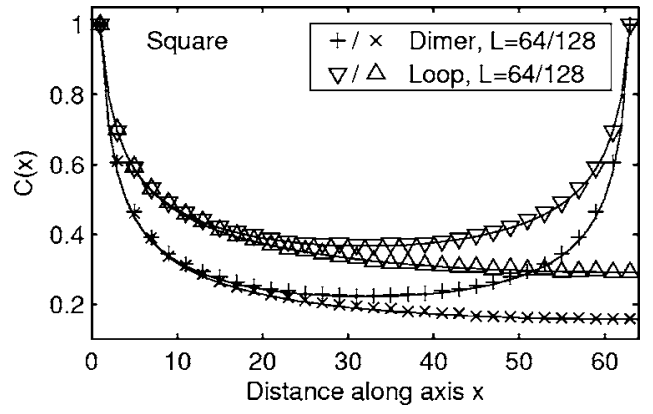

FIG. 3. Classical defect-defect correlation functions on a square lattice with hard-core dimer (upper panel) and loop dimer coverings (lower panel) along a coordinate axis. Results are shown for lattices with $L=64$ and $L=128$. The numerical data are fitted by the exact asymptotic results. The periodic boundary conditions are taken into account by plotting the fit against a rescaled distance variable.

which after normalization yields the correlation function $C(x)=Z(x) / Z_{0}$. The normalization is somewhat arbitrary; we chose it in such way that $C(1)=1$. The algorithm is terminated when the standard deviation of the measured quantity falls below a certain threshold. Results of simulations with different initial configurations are compared for verification. For the two-dimensional (2D) lattices about $10^{7}-10^{8}$ samplings were necessary. For the 3D diamond lattice it took about $10^{10}$ steps until convergence. We also applied an alternative Monte Carlo algorithm with loop updates ${ }^{14}$ which is known to be ergodic and unbiased but shows considerable slower convergence.

The algorithms have been applied to different lattice structures and filling factors (see Fig. 1). To test the implementation of the algorithm, we first reproduced the known monomer two-point correlations on the hard-core dimer covering on the square and triangular lattice (see Figs. 3 and 5) as well as the dipolar correlations in the undoped system on a square lattice $e^{14,15}$

The square lattice is a bipartite lattice and the two defects are on different sublattices. We extracted the exponent from the numerical data by linear interpolation of log-log plots and verified the results by finite-size scaling $C(x / L)$ $=L^{\gamma}(x / L)^{\gamma} c(x / L)$ with exponent $\gamma$ and system size $L$. Figure 3 compares the numerical data and the analytical results. For a rescaled distance variable $x^{\prime}=L \sin (\pi x / L) / \pi$ is used the numerical fit in order to account for the periodic boundary conditions.

In the case of a hard-core dimer covering (quarter-filled checkerboard lattice) the exponent agrees with the results from Ref. 16-i.e., $C(x) \sim 1 / x^{1 / 2}$. The correlations in the case of a loop dimer covering (half-filled checkerboard) are very well fitted with the power law $1 / x^{1 / 3}$, obtained analytically (see above). As expected, the correlations do not show any angle dependence at large distances. In both cases, the decay of the correlation function is algebraic which is expected for two-dimensional bipartite lattices: The two defects have long-range correlation and feel each other's presence at all distances. The bipartiteness is also seen by the strictly zero correlations $C(x)$ for distances which connect sites that reside in the same sublattice. 


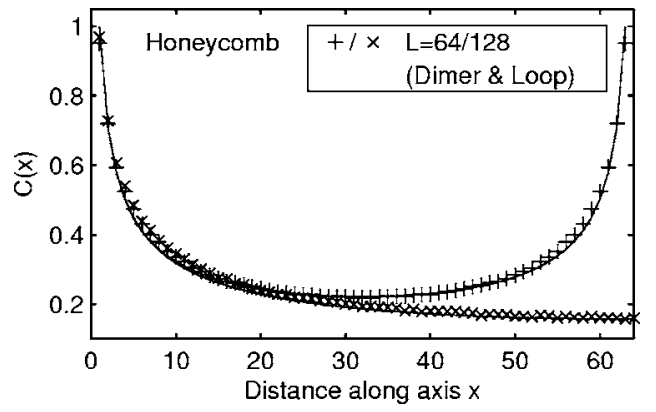

FIG. 4. Classical defect-defect correlation functions on a honeycomb lattice with hard-core dimer and loop dimer coverings along an axis of the bravais lattice. Numerical results are shown for lattices with $L=64$ and $L=128$ together with a fit to a power law $\sim x^{1 / 2}$. The periodic boundary conditions are taken into account by plotting the fit against a rescaled distance variable.

\section{B. Honeycomb lattice}

The kagomé lattices with nearest-neighbor repulsion at one-third and two-thirds filling are mapped to the hard-core dimer and the loop dimer model on the honeycomb lattice, respectively. The numerically obtained correlations along the axes of the bravais lattice are both $C(x) \sim 1 / x^{1 / 2}$ (see Fig. 4). Note that the two models-i.e., filling factors - are equivalent in the absence of defects. They can be identified by exchanging links which are occupied by a dimer and those which are not occupied. The faster algorithm described above did not show the equivalence of the two models. Therefore, we used the loop algorithm for this lattice.

\section{Triangular lattice}

The kagomé lattices with repulsion on the hexagons only is mapped at one-sixth filling to a hard-core dimer model and at one-third filling to a loop dimer model on the triangular lattice. The correlation function decays exponentially in both cases (see Fig. 5) with decay length of the order of one lattice spacing. The values obtained for the hard-core dimer model are in agreement with those obtained in Ref. 18. For distances farther than a few lattice spacings, $C(x)$ is constant within the noise ratio and tends to a finite value in the limit $x \rightarrow \infty .{ }^{19}$ This implies that the free energy difference $\Delta F(\infty)$

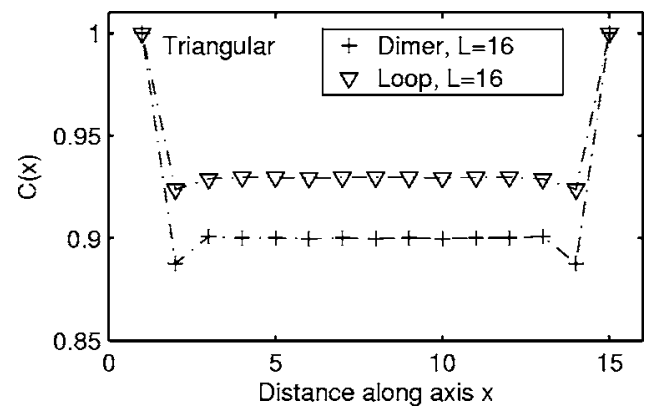

FIG. 5. Classical defect-defect correlation functions on a triangular lattice with hard-core dimer (upper panel) and loop dimer coverings (lower panel) along a coordinate axis. Results are shown for a lattice with $L=16$.

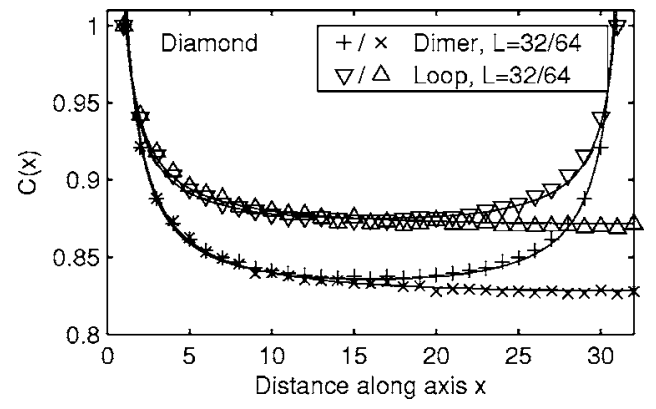

FIG. 6. Classical defect-defect correlation functions on a diamond lattice with hard-core dimer (upper panel) and loop coverings (lower panel) along a coordinate axis. Results are shown for lattices with $L=32$ and $L=64$. The numerical data are fitted to exponential functions. The periodic boundary conditions are taken into account by plotting the fit against a rescaled distance variable.

$\sim-T[\ln C(\infty)-\ln C(1)]$ of two infinitely separated defects in the classical hard-core dimer and loop dimer model is finite and the two defects are deconfined.

\section{Diamond lattice}

The mapping of particles with local constraints due to large repulsive interactions to hard-core dimer or loop-loop models can be applied to three-dimensional systems as well. For example, the pyrochlore lattices at one-fourth and one half-filling correspond to the hard-core dimer and the loop dimer model on the diamond lattice, respectively.

Monte Carlo simulations for two different lattice sizes are shown in Fig. 6. Much more samplings are needed to achieve a good signal-to-noise ratio, because the phase space is considerable larger than in the case of the 2D lattice. From a logarithmic plot, we found that the correlation functions decay approximately exponentially with respect to the inverse distance along the axes of the bravais lattice as $C(x) \sim \exp \left(\frac{1}{4 x}\right)$ for the hard-core dimer covering and $C(x)$ $\sim \exp \left(\frac{1}{6 x}\right)$ for the loop covering. The reason that this bipartite lattice shows these short-range correlations is because the monomers follow a 3D Coulomb law with potential $[V(r)$ $\sim 1 / r]$ as opposed to the divergence at large distances $V(r)$ $\sim \log r$ found in 2D. ${ }^{20}$ The restricted partition function then decays exponentially with distance in the case of the diamond lattice. A recent work on spin-1/2 Heisenberg antiferromagnets on pyrochlore lattices ${ }^{21}$ reveals a fractionalized spin liquid with $\mathrm{U}(1)$ gauge structure where this $1 / r$ potential acts between pairs of spinons and pairs of monopoles.

\section{DISCUSSION}

In summary, we calculated the classical defect-defect correlations for different fillings and different lattices with geometry that causes frustration in the motion of particles. (See Table I.) Recent studies on bipartite and nonbipartite threedimensional lattices ${ }^{20}$ lead to the conjecture that extended critical phases are realized only in bipartite lattices. This is supported by our results: For two-dimensional bipartitelattice-dual-lattice pairs (checkerboard-square lattice and 
TABLE I. The summary of the defect-defect correlations we obtained for the four different lattices and the two distinct dimer coverings. In the case of the triangular lattice there is an exponential decay with decay distance of one lattice constant, tending to a finite value at $x \rightarrow \infty$.

\begin{tabular}{ccc}
\hline \hline Lattice & Hard-core & Loop \\
\hline Square & $x^{-1 / 2}$ & $x^{-1 / 3}$ \\
Triangle & $\exp (-x)$ & $\exp (-x)$ \\
Honeycomb & $x^{-1 / 2}$ & $x^{-1 / 2}$ \\
Diamond & $\exp (1 / 4 x)$ & $\exp (1 / 6 x)$ \\
\hline \hline
\end{tabular}

kagomé-honeycomb lattice) the correlation functions decay with distance following a power law and tend to zero for $x$ $\rightarrow \infty$. Otherwise ["modified kagomé with repulsive interaction on hexagons only (triangular) lattice and three-dimensional pyrochlore (diamond) lattice"] an exponential decay with distance or inverse distance, respectively, and a finite value for $x \rightarrow \infty$ are found.

Where analytical values for power-law exponents were known in the literature, these could be confirmed. For the checkerboard lattice-i.e., two-dimensional pyrochlore lat- tice-we could relate the defect-defect correlations to the solved two-color fully packed loop model. ${ }^{13}$ This predicts $C(x) \sim x^{-1 / 3}$ which is in perfect agreement with the data from our Monte Carlo simulation.

The different behavior of the defect-defect correlations leads to two different scenarios with respect to the separation of defects at small finite temperatures. A separation of two defects to an infinite distance leads to an increase of the free energy, $\Delta F(\infty) \sim-T[\ln C(\infty)-\ln C(1)]$. Consequently, $\Delta F(\infty)$ is infinite for the defect on the square and honeycomb lattices (confinement) and remains finite for the triangular and diamond lattice (deconfinement).

The results are useful for the quantum version of the problem at the RK point of the Hamiltonian in general. ${ }^{7}$ In particular, our results can be extended to the motion of strongly correlated spinless fermions on the checkerboard lattice. ${ }^{8}$

\section{ACKNOWLEDGMENTS}

We are grateful to Peter Fulde, Kirill Shtengel, and Dima Efremov for useful discussions and in particular to Roderich Moessner for many discussions and valuable contributions to our understanding of classical correlations in dimer models.
*Present address: University of St. Andrews, North Haugh, St. Andrews KY16 9SS, Scotland, UK.

${ }^{1}$ P. W. Anderson, Science 235, 1196 (1987); also more recently L. Balents, M. P. A. Fisher, and S. M. Girvin, Phys. Rev. B 65, 224412 (2002) as relevant for quantum-frustrated antiferromagnetism.

${ }^{2}$ Frustrated Spin Systems, edited by H. T. Diep (World Scientific, Singapore, 2005).

${ }^{3}$ P. W. Anderson, Phys. Rev. 102, 1008 (1956).

${ }^{4}$ E. Runge and P. Fulde, Phys. Rev. B 70, 245113 (2004).

${ }^{5}$ P. Fulde, K. Penc, and N. Shannon, Ann. Phys. Leipzig 11, 892 (2002).

${ }^{6}$ M. E. Zhitomirsky, Phys. Rev. B 67, 104421 (2003).

${ }^{7}$ D. S. Rokshar and S. A. Kivelson, Phys. Rev. Lett. 61, 2376 (1988).

${ }^{8}$ F. Pollmann, J. Betouras, K. Shtengel, and P. Fulde (unpublished).

${ }^{9}$ R. H. Fowler and G. S. Rushbrooke, Trans. Faraday Soc. 33, 1272 (1937).

${ }^{10}$ P. W. Kasteleyn, Physica (Amsterdam) 27, 1209 (1961).
${ }^{11}$ M. E. Fisher and J. Stephenson, Phys. Rev. 132, 1411 (1963).

${ }^{12}$ R. E. Hartwig, J. Math. Phys. 7, 286 (1966).

${ }^{13}$ J. Kondev and J. L. Jacobsen, Phys. Rev. Lett. 81, 2922 (1998); J. L. Jacobsen and J. Kondev, Nucl. Phys. B 532, 635 (1998).

${ }^{14}$ R. Moessner, O. Tchernyshyov, and S. L. Sondhi, J. Stat. Phys. 116, 755 (2004).

${ }^{15}$ S. V. Isakov, K. Gregor, R. Moessner, and S. L. Sondhi, Phys. Rev. Lett. 93, 167204 (2004).

${ }^{16}$ W. Krauth and R. Moessner, Phys. Rev. B 67, 064503 (2003).

${ }^{17}$ O. F. Syljuasen and A. W. Sandvik, Phys. Rev. E 66, 046701 (2002).

${ }^{18}$ P. Fendley, R. Moessner, and S. L. Sondhi, Phys. Rev. B 66, 214513 (2002).

${ }^{19}$ R. Moessner and S. L. Sondhi, Phys. Rev. Lett. 86, 1881 (2001).

${ }^{20}$ D A. Huse, W. Krauth, R. Moessner, and S. L. Sondhi, Phys. Rev. Lett. 91, 167004 (2003).

${ }^{21}$ M. Hermele, M. P. A. Fisher, and L. Balents, Phys. Rev. B 69, 064404 (2004). 